\title{
Conductance fluctuations in a quantum dot under almost periodic ac pumping.
}

\author{
Xiao-Bing Wang $^{1}$ and V. E. Kravtsov ${ }^{1,2}$ \\ 1 Tha Abdus Salam International Centre for Theoretical Physics, P.O. Box 586, 34100 Trieste, Italy \\ ${ }^{2}$ Landau Institute for Theoretical Physics, 2 Kosygina Street, 117940 Moscow, Russia
}

(October 31, 2018)

\begin{abstract}
It is shown that the variance of the linear dc conductance fluctuations in an open quantum dot under a high-frequency ac pumping depends significantly on the spectral content of the ac field. For a sufficiently strong ac field $\gamma \tau_{\varphi} \ll 1$, where $1 / \tau_{\varphi}$ is the dephasing rate induced by ac noise and $\gamma$ is the electron escape rate, the dc conductance fluctuations are much stronger for the harmonic pumping than in the case of the noise ac field of the same intensity. The reduction factor $r$ in a static magnetic field takes the universal value of 2 only for the white-noise pumping. For the strictly harmonic pumping $A(t)=A_{0} \cos \omega t$ of sufficiently large intensity the variance is almost insensitive to the static magnetic field $r-1=2 \sqrt{\tau_{\varphi} \gamma} \ll 1$. For the quasi-periodic ac field of the form $A(t)=A_{0}\left[\cos \left(\omega_{1} t\right)+\cos \left(\omega_{2} t\right)\right]$ with $\omega_{1,2} \gg \gamma$ and $\gamma \tau_{\varphi} \ll 1$ we predict the novel effect of enchancement of conductance fluctuations at commensurate frequencies $\omega_{2} / \omega_{1}=P / Q$.

PACS numbers: 72.15.-v, 72.30.+q, 73.23.-b
\end{abstract}

One of the most important discoveries in mesoscopic physics is the universal conductance fluctuations [1,2. These fluctuations of the order of $\delta G \sim e^{2} / \hbar$, are due to stochastic quantum interference. They depend only on the effective dimensionality and general symmetries, but not on the microscopic details of the system. The fluctuations can manifest themselves as reproducible aperiodic magneto-resistance patterns(magnetic fingerprints). Variation of the fluctuations with magnetic fields and chemical potential, temperature etc. were calculated by the diagrammatic technique [3] and observed in many disordered electronic systems 4 . In the presence of a strong enough static magnetic field, the time reversal symmetry is broken and the variance $\left\langle\delta G^{2}\right\rangle(\langle\ldots\rangle$ stands for the disorder average) of conductance fluctuations is reduced by the factor $r=2$.

Recently there has been a considerable interest in nonequilibrium mesoscopics. The effect of adiabatic charge pumping [5] has been experimentally observed [6] and analyzed theoretically [7,8]. Weak localization under ac pumping [9] and the photovoltaic effect [12] in a quantum dot have been theoretically studied. The non-equilibrium noise has been suggested [15] as a cause of both the low temperature dephasing saturation [13] and the anomalously large ensemble averaged persistent current 14.

Here we study the effect of the high-frequency ac field on the mesoscopic fluctuations of linear dc conductance when both the weak dc voltage and a strong enough highfequency pump field are applied to the open quantum dot [16]. We will study the dependence of the variance of the dc conductance fluctuations on the ac field intensity for the noise-like and an almost periodic ac field. In particular we focus on the reduction factor $r=1+\mathcal{C} / \mathcal{D}$ for the variance of conductance fluctuations after turning on a strong time-reversal breaking (e.g. the static magnetic field) that kills the cooperon contribution to the variance $\left\langle\delta G^{2}\right\rangle_{C} \equiv \mathcal{C}$ while leaving the diffuson one $\left\langle\delta G^{2}\right\rangle_{D} \equiv \mathcal{D}$ unchanged.

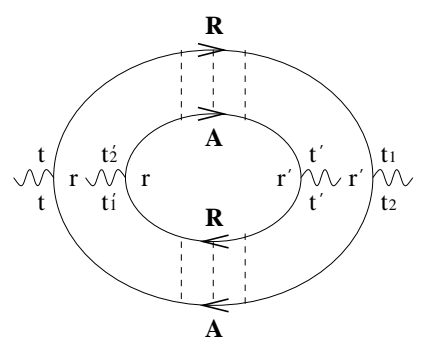

(a)

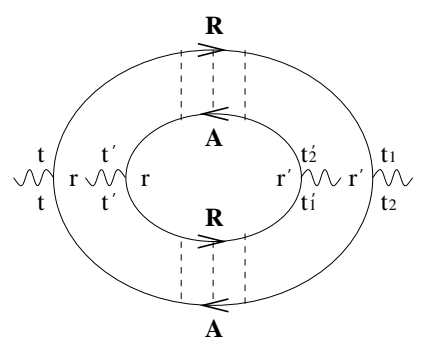

(b)
FIG. 1. The cooperon (a) and the diffuson (b) contributions to the variance of conductance fluctuations.

The Landauer conductance $g=(\gamma V / 4)^{2}\left[K\left(\mathbf{r}, \mathbf{r}^{\prime}\right)+\right.$ $\left.K\left(\mathbf{r}^{\prime}, \mathbf{r}\right)\right]$ of a dot of the volume $V$ with small contacts at $\mathbf{r}$ and $\mathbf{r}^{\prime}$ and the electron escape rate $\gamma$, can be expressed in terms of the exact retarded and advanced electron Green's functions $G^{R, A}\left(\mathbf{r}, \mathbf{r}^{\prime} ; t, t^{\prime}\right)$ in the time domain [9]10]:

$K\left(\mathbf{r}, \mathbf{r}^{\prime}\right)=\int d t_{1} d t_{2} \overline{G^{R}\left(\mathbf{r}, \mathbf{r}^{\prime} ; t, t_{1}\right) G^{A}\left(\mathbf{r}^{\prime}, \mathbf{r} ; t_{2}, t\right)} F_{t_{1}-t_{2}}$

where $F_{t}=\pi T t \sinh ^{-1}(\pi T t)$ is the Fourier-transform of the derivative of the Fermi distribution function for electrons in the leads and $\overline{f(t)}=\int_{-\mathcal{T} / 2}^{\mathcal{T} / 2} \frac{d t}{\mathcal{T}} f(t)$ denotes time averaging during the observation time $\mathcal{T} \rightarrow \infty$.

We consider a chaotic or disordered quantum dot with the number of open channels $M \gg 1$ and the electron escape rate $\Delta \ll \gamma=\Delta M \ll E_{c}$ where $\Delta$ is the mean level separation and the Thouless energy $E_{c}$ is the inverse ergodic time. In this situation the charging effects are negligible, the perturbative diagrammatic analysis (see Fig.1) is possible and the ergodic zero-dimensional approximation applies. We also assume the dephasing rate $\gamma_{\text {int }}(T)$ caused by electron interaction to be smaller than the escape rate $\gamma$. At such conditions in the absence of ac pumping one obtains $\mathcal{C}=\mathcal{D} \sim(\gamma / \Delta)^{2}$ at zero temperature. Thus the reduction factor $r=2$. 
However, the results may change if the dot is subject to a time-dependent perturbation. In that case the Green's functions $G^{R, A}\left(\mathbf{r}, \mathbf{r}^{\prime} ; t, t^{\prime}\right)$ are inhomogeneous in time, so that the cooperon

$$
\left\langle G^{R}\left(\mathbf{r}, \mathbf{r}^{\prime} ; t_{+}, t_{+}^{\prime}\right) G^{A}\left(\mathbf{r}, \mathbf{r}^{\prime} ; t_{-}, t_{-}^{\prime}\right)\right\rangle=\frac{1}{2} \delta\left(t-t^{\prime}\right) C_{t}\left(\eta, \eta^{\prime}\right)
$$

and the diffuson

$$
\left\langle G^{R}\left(\mathbf{r}, \mathbf{r}^{\prime} ; t_{+}, t_{+}^{\prime}\right) G^{A}\left(\mathbf{r}^{\prime}, \mathbf{r} ; t_{-}^{\prime}, t_{-}\right)\right\rangle=\delta\left(\eta-\eta^{\prime}\right) D_{\eta}\left(t, t^{\prime}\right)
$$

are no longer functions of the difference of two times but in general depend on four time variables $t_{ \pm}=$ $t \pm \eta / 2, t_{ \pm}^{\prime}=t^{\prime} \pm \eta^{\prime} / 2$. They can be found from [18]:

$$
\begin{aligned}
\left\{2 \frac{\partial}{\partial \eta}+\gamma+\right. & \left.D\left[i \nabla+A\left(t+\frac{\eta}{2}\right)+A\left(t-\frac{\eta}{2}\right)\right]^{2}\right\} \\
& \times C_{t}\left(\eta, \eta^{\prime} ; \mathbf{r}, \mathbf{r}^{\prime}\right)=2 \delta\left(\eta-\eta^{\prime}\right) \delta\left(\mathbf{r}-\mathbf{r}^{\prime}\right),
\end{aligned}
$$

and

$$
\begin{aligned}
\left\{\frac{\partial}{\partial t}+\gamma+D\right. & {\left.\left[i \nabla+A\left(t+\frac{\eta}{2}\right)-A\left(t-\frac{\eta}{2}\right)\right]^{2}\right\} } \\
& \times D_{\eta}\left(t, t^{\prime} ; \mathbf{r}, \mathbf{r}^{\prime}\right)=\delta\left(t-t^{\prime}\right) \delta\left(\mathbf{r}-\mathbf{r}^{\prime}\right)
\end{aligned}
$$

For concreteness we consider the 'dot' in a form of a quasi-1d ring of the circumference $L$ with two leads at points $\mathbf{r}$ and $\mathbf{r}^{\prime}$. Qualitatively, the results do not change if the dot has the form of a disc or the ac pumping is produced by applying the gate voltage that changes the dot's shape 11].

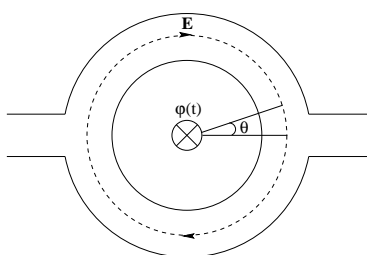

FIG. 2. The circular 'dot' under ac pumping: the circular electric field results from the oscillating magnetic flux $\varphi(t)$.

The time-dependent magnetic flux $\phi(t)=A(t) L$ threads the ring and results in the circular electric field $E_{\theta}(t)=-d / d t A(t)$ which does not depend on the position $x=L \theta / 2 \pi$ (see Fig.2). The periodic boundary condition $D_{\eta}\left(t, t^{\prime} ; x-x^{\prime}\right)=D_{\eta}\left(t, t^{\prime} ; x+L-x^{\prime}\right)$ and $C_{t}\left(\eta, \eta^{\prime} ; x-x^{\prime}\right)=C_{t}\left(\eta, \eta^{\prime} ; x+L-x^{\prime}\right)$ allows to switch in Eqs. (2, 3) to the Fourier components $D_{\eta}\left(t, t^{\prime} ; q\right)$ and $C_{t}\left(\eta, \eta^{\prime} ; q\right)$ with $q_{n}=(2 \pi / L) n,(n=0, \pm 1, \pm 2, \ldots)$ and the condition $E_{c} / \gamma \gg 1$ allows to leave only the zero-mode components $D_{\eta}\left(t, t^{\prime}\right)=D_{\eta}\left(t, t^{\prime} ; q=0\right)$ and $C_{t}\left(\eta, \eta^{\prime}\right)=C_{t}\left(\eta, \eta^{\prime} ; q=0\right)$ (ergodic approximation):

$$
\begin{gathered}
D_{\eta}\left(t, t^{\prime}\right)=\Theta_{\eta-\eta^{\prime}} e^{-\int_{t^{\prime}}^{t} \Gamma_{d}(\eta, \xi) d \xi} \\
C_{t}\left(\eta, \eta^{\prime}\right)=\Theta_{t-t^{\prime}} e^{-\frac{1}{2} \int_{\eta^{\prime}}^{\eta} \Gamma_{c}(t, \xi) d \xi},
\end{gathered}
$$

where $\Theta_{t}$ is the step-function and [11]:

$$
\begin{gathered}
\Gamma_{d}(\eta, \xi)=\gamma+D(A(\xi+\eta / 2)-A(\xi-\eta / 2))^{2} \\
\Gamma_{c}(t, \xi)=\gamma+D(A(t+\xi / 2)+A(t-\xi / 2))^{2} .
\end{gathered}
$$

Then one obtains from the diagrams of Fig.1:

$$
\mathcal{D} \propto \int d t^{\prime} d t^{\prime \prime} \overline{D_{\eta}\left(t, t^{\prime}\right) D_{-\eta}\left(t, t^{\prime \prime}\right)} F_{t^{\prime}-t^{\prime \prime}}^{2}
$$

$\mathcal{C} \propto \int d t^{\prime} d \eta^{\prime} \overline{C_{t^{\prime}+t}\left(-\eta^{\prime}-2 t^{\prime},-\eta+2 t^{\prime}\right) C_{t}\left(\eta, \eta^{\prime}\right)} F_{2 t^{\prime}}^{2}$

where averaging over $t$ and $\eta$ is assumed.

We observe that while the variables in the arguments of diffusons are decoupled, they are strongly coupled in the argument of a cooperon. As usual this leads to the restricted region of integration in Eq.(9) and to a general inequality $\mathcal{C} / \mathcal{D}<1$ or $r<2$.

However, there is a dramatic difference between the noise-like ac field with the short correlation time $\tau_{0} \sim$ $\omega^{-1} \ll \tau_{\varphi}$ and the harmonic ac field $A(t)=A_{0} \cos (\omega t)$ with $\omega \gg \gamma$. At such a high-frequency pumping one can replace $\Gamma_{d}(\eta, \xi)$ and $\Gamma_{c}(t, \xi)$ in Eqs. (4,5) by the time averages $\Gamma_{d}(\eta)=\overline{\Gamma_{d}(\eta, \xi)}$ and $\Gamma_{c}(t)=\overline{\Gamma_{c}(t, \xi)}$. This approximation will be referred to as the high frequency ansatz. In the case of the white-noise pumping the average of the cross-terms $\overline{A_{\xi+\eta / 2} A_{\xi-\eta / 2}}$ and $\overline{A_{t+\xi / 2} A_{t-\xi / 2}}$ in Eqs.(6,7) is zero and we obtain the same, time-independent decay rates $\Gamma_{d}=\Gamma_{c}=\gamma+1 / 2 \tau_{\varphi}$ for the cooperons and the diffusons with $\eta \neq 0$, where

$$
\tau_{\varphi}^{-1}=4 D \overline{A^{2}} \propto I
$$

is proportional to the pumping intensity $I$. As the result the reduction factor $r=2$ is unchanged. In contrast to that, for the harmonic pumping the decay rates $\Gamma_{c}(t)=\gamma+\tau_{\varphi}^{-1} \cos ^{2}(\omega t)$ and $\Gamma_{d}(\eta)=\gamma+\tau_{\varphi}^{-1} \sin ^{2}(\omega \eta / 2)$ are periodic functions of the 'additional' time variables $t$ and $\eta$. Thus the pumping-induced dephasing is effectively switched off inside the 'no-dephasing' time intervals where $\Gamma_{d, c}(t) \ll \gamma$. At strong pumping $\tau_{\varphi}^{-1} \gg \gamma$ these time intervals make the main contribution to mesoscopic fluctuations.

Below we present analytical results for the reduction factor $r$ in the high-frequency limit $\omega \gg \tau_{\varphi}^{-1} \gg \gamma$ 11.

Using the high-frequency ansatz we obtain from Eqs. (8,9):

$$
\begin{gathered}
\frac{\mathcal{D}}{g_{0}^{2}}=\int_{-\mathcal{T} / 2}^{\mathcal{T} / 2} \frac{d \eta}{2 \mathcal{T}}\left(\frac{\gamma^{2}}{\Gamma_{d}(\eta)}\right) \int_{0}^{\infty} e^{-t \Gamma_{d}(\eta)} F_{t}^{2} d t . \\
\frac{\mathcal{C}}{g_{0}^{2}}=\int_{-\mathcal{T} / 2}^{\mathcal{T} / 2} \frac{d t}{\mathcal{T}} \int_{-\infty}^{t} d t^{\prime} \frac{\gamma^{2} F_{2 t-2 t^{\prime}}^{2} e^{-2\left(t-t^{\prime}\right) \Gamma_{c}\left(t^{\prime}\right)}}{\frac{1}{2}\left(\Gamma_{c}(t)+\Gamma_{c}\left(t^{\prime}\right)\right)},
\end{gathered}
$$


where $g_{0}=\pi \gamma / 4 \Delta$ is the mean conductance, $\mathcal{T} \rightarrow \infty$ is an observation time, and $F_{t}=\pi T t \sinh ^{-1}(\pi T t)$.

Eqs.(11.12) can be simplified in the limit of low temperatures $T \ll \gamma$ where $F_{t} \approx 1$ and in the limit of high temperatures $T \gg \omega\left(\gamma \tau_{\varphi}\right)^{-1 / 2}$ where $F_{t}^{2} \approx(\pi / 6 T) \delta(t)$ and we have:

$$
\frac{\left\langle\delta G^{2}\right\rangle_{D, C}}{g_{0}^{2}}=\frac{\pi \gamma^{2}}{12 T} \int_{-\mathcal{T} / 2}^{\mathcal{T} / 2} \frac{d t}{\mathcal{T}} \frac{1}{\Gamma_{d, c}(t)} .
$$

As has been already mentioned, in the case of the nearly white-noise pumping $\Gamma_{d}=\Gamma_{c}=\gamma+1 / 2 \tau_{\varphi}$ are independent of $\eta$ and $t$. For relatively small pumping intensity $I \propto\left(\gamma \tau_{\varphi}\right)^{-1} \ll \gamma / \Delta$ the variance of conductance fluctuations is still given by diagrams of Fig.1 and we have:

$$
\frac{\mathcal{D}}{g_{0}^{2}}=\frac{\mathcal{C}}{g_{0}^{2}}=\left\{\begin{array}{cl}
\frac{1}{2}\left(1+\frac{1}{2 \gamma \tau_{\varphi}}\right)^{-2}, & T \ll \gamma+\frac{1}{2 \tau_{\varphi}} \\
\frac{\pi \gamma}{12 T}\left(1+\frac{1}{2 \gamma \tau_{\varphi}}\right)^{-1}, & T \gg \gamma+\frac{1}{2 \tau_{\varphi}}
\end{array}\right.
$$

For larger intensities $\left(\gamma \tau_{\varphi}\right)^{-1} \gg \gamma / \Delta$ one has to take into account dressing of vertices 117 and the result changes. Yet in both cases the reduction factor $r$ is strictly 2 .

For the periodic pumping the decay rates are periodic functions of time: $\Gamma_{d}(t)=\gamma+\tau_{\varphi}^{-1} \sin ^{2}(\omega t / 2)$, $\Gamma_{c}(t)=\gamma+\tau_{\varphi}^{-1} \cos ^{2}(\omega t)$, and Eqs.(11.12) no longer lead to the same result. At high pumping intensities $\gamma \tau_{\varphi} \ll 1$ the integrals in Eqs.(11) 12) are dominated by the vicinity of zeros $t_{n}^{D, C}$ of $\sin ^{2}(\omega t / 2)$ and $\cos ^{2}(\omega t)$. One can expand $\Gamma_{d, c}(t)$ near $t_{n}^{D, C}$, perform integrations from $-\infty$ to $+\infty$ over $t-t_{n}^{D, C}$ and sum over $t_{n}^{D, C}$. The result depends on the relation between the temperature of leads $T$ and $\gamma$.

For $T \ll \gamma \ll \tau_{\varphi}^{-1}$ we obtain:

$$
\frac{\mathcal{D}}{g_{0}^{2}} \approx \frac{1}{4} \sqrt{\gamma \tau_{\varphi}} \propto \frac{1}{\sqrt{I}}, \quad \frac{\mathcal{C}}{g_{0}^{2}} \approx \frac{1}{2} \gamma \tau_{\varphi} \propto \frac{1}{I} .
$$

One can see that the cooperon contribution $\mathcal{C}$ is strongly suppressed at high pumping intensities and the reduction factor $r=1+2\left(\gamma \tau_{\varphi}\right)^{1 / 2}$ is close to 1 .

At high temperatures $T \gg \omega\left(\gamma \tau_{\varphi}\right)^{-1 / 2}$ and strong pumping $\gamma \ll \tau_{\varphi}^{-1}$ Eq. (13) gives the same value

$$
\frac{\mathcal{D}}{g_{0}^{2}}=\frac{\mathcal{C}}{g_{0}^{2}}=\frac{\pi \gamma}{12 T} \sqrt{\gamma \tau_{\varphi}}
$$

for the diffuson and the cooperon contribution to conductance fluctuations, and $r=2$.

However, even at high temperatures the cooperon contribution can be significantly suppressed relative to the diffuson one if the pumping field is a sum of two harmonic parts:

$$
A(t)=A_{0}[\cos (\omega t)+\cos (\alpha \omega t)], \quad(0<\alpha<1) .
$$

In this case we have from Eqs.(6.7.7) $\Gamma_{d}(t)-\gamma=$ $\tau_{\varphi}^{-1}\left[\sin ^{2}(\omega t / 2)+\sin ^{2}(\alpha \omega t / 2)\right] / 2$ and $\Gamma_{c}(t)-\gamma=$ $\tau_{\varphi}^{-1}\left[\cos ^{2}(\omega t)+\cos ^{2}(\alpha \omega t)\right] / 2$. In the limit of strong pumping $\gamma \tau_{\varphi} \ll 1$ one can express the integral in Eq. (13) in terms of the distribution of complex roots $t_{n}$ of the equation $\Gamma_{d, c}(t)=\gamma$. This is the problem of distribution of complex roots $z_{n}=x_{n}+i y_{n}$ with $y_{n} \sim \sqrt{\gamma \tau_{\varphi}} \ll 1$ of the transcendental equation:

$$
\cos z+\cos (\alpha z) \pm 2=0,
$$

where the sign \pm stands for the cooperon and the diffuson contribution to the variance, respectively.

Defining the densities of complex roots $\rho_{d, c}(y)$ of Eq.(18) with the sign $-\left(\rho_{d}\right)$ or $+\left(\rho_{c}\right)$ :

$$
\rho(y)=\sum_{n}\left\langle\delta\left(x-x_{n}\right) \delta\left(y-y_{n}\right)\right\rangle_{x},
$$

where $\langle\ldots\rangle_{x}=(\omega \mathcal{T})^{-1} \int_{|x|<\omega \mathcal{T} / 2} d x \ldots$ stands for the averaging over $x$, we obtain:

$$
\frac{\left\langle\delta G^{2}\right\rangle_{D, C}}{g_{0}^{2}}=\frac{\pi^{2} \gamma^{2} \tau_{\varphi}}{3 T \sqrt{1+\alpha^{2}}} \int_{-\infty}^{+\infty} \frac{d y \rho_{d, c}(y)}{\left[2 \gamma \tau_{\varphi}+\frac{y^{2}}{4}\left(1+\alpha^{2}\right)\right]^{1 / 2}} .
$$

For the case of two commensurate frequencies $\alpha=P / Q<$ 1 with $Q \sim 1$ the density $\rho(y)$ is the set of $\delta$-functions $(2 \pi Q)^{-1} \sum \delta\left(y-y_{n}\right)$ separated by gaps $\Delta y_{n} \sim 1 / Q$. At $\gamma \tau_{\varphi} \ll 1 / Q^{2}$ the gap $y_{1} \sim 1 / Q$ is large compared to $\left(\gamma \tau_{\varphi}\right)^{1 / 2}$, and in the leading approximation one can neglect all complex roots with $y_{n} \neq 0$. Equation (18) with the sign minus has real roots at any rational $\alpha$. However, Eq.(18) with the sign plus (relevant for the cooperon contribution) has real solutions only if $P$ and $Q$ are both odd. In this case $\mathcal{D}=\mathcal{C} \propto I^{-1 / 2}$ as for a strictly harmonic pumping. However the cooperon contribution is anomalously suppressed $\mathcal{C} \propto I^{-1}$ if either $Q$ or $P$ is even. This parity effect is also present for $T \ll \gamma$ as it is seen from Fig.3 obtained by numerical integration of Eqs.(11, 12).

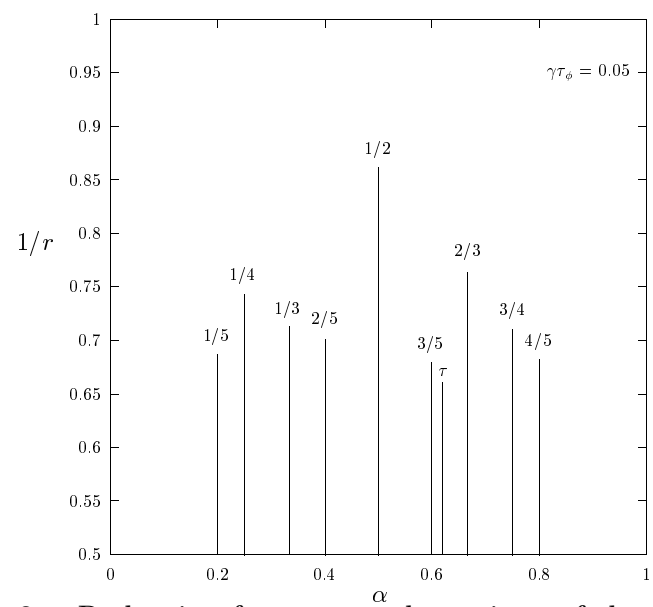

FIG. 3. Reduction factor $r$ vs the ratio $\alpha$ of the two frequency components. Also plotted is $r$ for the golden mean $\tau=\frac{\sqrt{5}-1}{2}$. 
With increasing $Q$ the gaps $\Delta y_{n}$ shrink to zero and in the limit $Q \rightarrow \infty$ one obtains smooth distribution $\rho(y)$ which have a deep number-theoretical origin. The behavior of $\rho(y)$ at small $y$ determines the dependence of $\mathcal{D}$ and $\mathcal{C}$ on the pumping intensity $I \propto\left(\gamma \tau_{\varphi}\right)^{-1}$.

For an infinite observation time $\mathcal{T} \rightarrow \infty$ the function $\rho(y)$ is discontinuous at any commensurate point $\alpha=P / Q$. For instance at $\alpha=1 / 2$ we have $\rho_{d}(y)=$ $(4 \pi)^{-1} \delta(y)$ and $\rho_{c}(y)=0$ at $y \ll 1$. However at any $\alpha \rightarrow 1 / 2$ the smooth distribution $\rho_{d}(y)=\rho_{c}(y)$ does not show any peak at $y=0$. This means that at a strong pumping the diffuson part of the variance of conductance fluctuations drops dramatically if one goes away from $\alpha=1 / 2$ and simultaneously the reduction factor moves towards the universal value. This variation is discontinuous at $\mathcal{T} \rightarrow \infty$ but at a finite observation time $\mathcal{T}$ (finite width $\delta \sim 1 / \mathcal{T}$ of the harmonic component) it happens at a scale $\delta \alpha=\delta / \omega$. This is illustrated by Fig.4 obtained by the numerical evaluation of Eqs.(11),(12) at zero temperature.

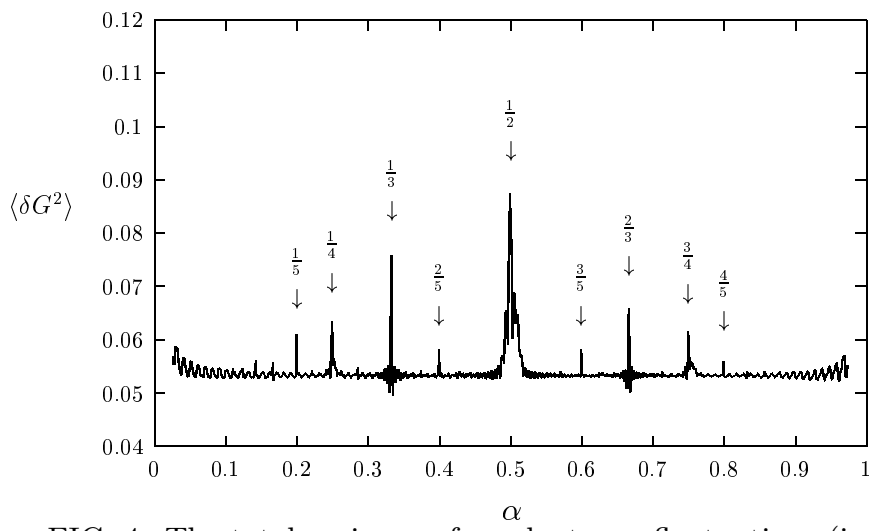

FIG. 4. The total variance of conductance fluctuations (in units of the ensemble-average dc conductance) as a function of $\alpha$ at $\gamma \tau_{\phi}=0.05$ and $1 / \mathcal{T} \omega=0.01$. The width of peaks is of the order of $1 / \mathcal{T} \omega$.

In conclusion, we have derived expressions for the diffuson and the cooperon contributions to the variance of conductance fluctuations in an open quantum dot subject to ac pumping. We focus at the difference between the white-noise and almost periodic ac pumping. We show that in the case of an almost periodic ac field the pumping-induced dephasing is effectively switched off inside the 'no-dephasing' time windows. That is why the periodic ac field is much less effective in the suppression of conductance fluctuations than the noise-like ac field. In contrast to the ac noise, the periodic ac field may lead to the deviation of the reduction factor $r$ (in the presence of a strong phase-breaking mechanism) from the universal value of 2 . The dependence of the variance of conductance fluctuations on the pumping intensity is studied for the harmonic pumping and the quasi-periodic pumping with two harmonic components. In the latter case we predict the new effect of enchancement of conductance fluctuations if two frequencies are commensurate.

We are grateful to I.L.Aleiner and I.V.Lerner for a clarifying discussion and especially to E.Kanzieper and V.I.Yudson for a fruitful exchange of ideas during collaboration on a similar problem.

[1] R. A. Webb, S. Washburn, C. P. Umbach, and R. B. Laibowitz, Phys. Rev. Lett. 54, 2692(1985).

[2] B. L. Altshuler, Pis'ma Zh. Eksp. Teor. Fiz. 51, 530(1985)[JETP Lett. 41, 648(1985)]; P. A. Lee and A. D. Stone, Phys. Rev. Lett. 55, 1622(1985).

[3] B. L. Altshuler and D. E. Khmelniskii, Pis'ma Zh. Eksp. Teor. Fiz. 42, 291(1985)[JETP Lett. 42, 359(1985)].

[4] N. Giordano, in Mesoscopic Phenomena in Solids, edited by B. L. Altshuler, P. A. Lee and R. A. Webb(Elsevier, North-Holand, 1991).

[5] D. J. Thouless, Phys. Rev. B 27, 6083 (1983).

[6] M. Switkes, C. M. Marcus, K. Campman, A. C. Grossard, Science 283, 1905 (1999).

[7] P. W. Brouwer, Phys. Rev. B 58, 10135 (1998); F. Zhou et al. Phys. Rev. Lett., 82, 608 (1999).

[8] T. A. Shutenko, I. L. Aleiner, and B. L. Altshuler, Phys. Rev. B 61, 10366 (2000).

[9] M. G. Vavilov and I. L. Aleiner, Phys. Rev. B, 60, R16311 (1999).

[10] V.I.Yudson, E.Kanzieper and V.E.Kravtsov, condmat/0012200.

[11] In the experimental geometry shown in Fig.2 there is no restriction connected with the relationship between the pumping frequency $\omega$ and the Thouless energy $E_{c}$. However, if pumping is implemented by changing of the dot shape or by the longitudinal time-dependent electric field $E(t)$, the boundary conditions set the constraint on the applicability of Eqs. (4.7). They are valid only for $\omega \gg E_{c}\left[10\right.$. For $\omega \ll E_{c}$ one can derive $\Gamma_{d}(\eta, \xi)-\gamma=C(E(\xi+\eta / 2)-E(\xi-\eta / 2))^{2}$ and $\Gamma_{c}(t, \xi)-\gamma=(C / 4)(E(t+\xi / 2)-E(t-\xi / 2))^{2}$ with $C \sim L^{2} / E_{c} 12,10$. This leads only to the re-definition of the parameter $\tau_{\varphi}^{-1}$ in Eq. $(10): \tau_{\varphi}^{-1}=4 C \overline{E^{2}}$.

[12] M. G. Vavilov, V. Ambegaokar and I. Aleiner, condmat/0008469.

[13] P. Mohanty, E. M. Q. Jariwala, and R. A. Webb, Phys. Rev. Lett. 78, 3366(1997).

[14] L. P. Levi, G. Dolan, J. Dunsmuir, and H. Bouchiat, Phys. Rev. Lett. 64, 2074 (1990).

[15] V. E. Kravtsov and B. L. Altshuler, Phys. Rev. Lett. 84, 3394(2000).

[16] for a weak pumping this problem has been first considered in: V.I.Falko, Europhys Lett. 8, 785 (1989).

[17] E. MaCann ang I. V. Lerner, Phys. Rev. B 57, 7219(1998).

[18] B. L. Altshuler, A. G. Aronov, and D. E. Khmelniskii, J. Phys. C 15, 7367(1982). 